Hiromichi Fujiwara • Mitsuru Emi • Hisaki Nagai

Taiji Nishimura • Noboru Konishi • Yoshinobu Kubota

Tomohiko Ichikawa - Satoru Takahashi - Taro Shuin

Tomonori Habuchi · Osamu Ogawa · Katsuki Inoue

Mark H. Skolnick • Jeff Swensen • Nicola J. Camp

Sean V. Tavtigian

\title{
Association of common missense changes in ELAC2 (HPC2) with prostate cancer in a Japanese case-control series
}

Received: September 3, 2002 / Accepted: October 2, 2002

\begin{abstract}
The recently identified prostate cancer susceptibility gene ELAC2 (HPC2) harbors two common missense variants, a serine to leucine substitution at residue 217 (Leu217) and an alanine to threonine substitution at residue 541 (Thr541). We genotyped the two variants in a Japanese cohort consisting of 350 prostate cancer patients 242 male
\end{abstract}

H. Fujiwara $\cdot$ M. Emi $(\bowtie) \cdot$ H. Nagai

Department of Molecular Biology, Institute of Gerontology, Nippon Medical School, 1-396 Kosugi-cho, Nakahara-ku, Kawasaki 211-8533, Japan

Tel. +81-44-733-5230; Fax +81-44-733-5192

e-mail: memi@nms.ac.jp

H. Fujiwara $\cdot$ T. Nishimura

Department of Urology, Nippon Medical School, Kawasaki, Japan

N. Konishi

Department of Pathology, Nara Medical University, Nara, Japan

Y. Kubota

Department of Urology, Yokohama City University Medical School, Yokohama, Japan

T. Ichikawa

Department of Urology, Graduate School of Medicine, Chiba

University, Chiba, Japan

S. Takahashi

Department of Pathology, Nagoya City University Medical School,

Nagoya, Japan

T. Shuin

Department of Urology, Kouchi Medical University, Kouchi, Japan

T. Habuchi

Department of Urology, Akita University Medical School, Akita,

Japan

O. Ogawa

Department of Urology, University of Kyoto Medical School, Kyoto, Japan

K. Inoue

University of Hawaii, Queen's Medical Center, Honolulu, HI, USA

M.H. Skolnick · J. Swensen · S.V. Tavtigian

Myriad Genetics, Inc., Salt Lake City, UT, USA

N.J. Camp

Genetic Epidemiology Group, Department of Medical Informatics,

University of Utah School of Medicine, Salt Lake City, UT, USA population controls, and 114 male low-risk controls. Both missense alleles, Leu217 and Thr541, were carried at higher frequency in Japanese patients than in the controls (Leu217, $P=0.0012$; Thr541, $P=0.0145$ ), and the odds ratios associated with carrying these sequence variants were higher in Japanese than in Caucasians. Although the Leu217 and Thr541 variants of ELAC2 are less common in Japanese than in Caucasians, both variants confer significantly increased risk of prostate cancer in Japanese. Carriage of these variants was not associated with age at diagnosis, tumor stage, or tumor grade in these Japanese prostate cancer patients. The allele-specific pattern of risk observed in Japanese and familial Caucasian patients was qualitatively similar; however, the magnitude of that risk was considerably greater in Japanese than in Caucasians.

Key words $E L A C 2 \cdot H P C 2 \cdot$ Modest risk variant $\cdot$ Prostate cancer $\cdot$ Genetics

\section{Introduction}

ELAC2 (HPC2), the first prostate cancer susceptibility gene identified through genetic linkage and positional cloning, was described in late 2000 (Tavtigian et al. 2000). Orthologs of ELAC2 are readily identifiable among all eukaryotes for which complete genome sequences are available. In addition, the carboxy half of $E L A C 2$ is very similar to a gene, typified by Escherichia coli elaC, present in all prokaryotes. Sequence analysis suggests that ELAC2 encodes a metal-dependent hydrolase. However, the function of the gene product remains unknown, as does the mechanism by which the altered function influences the risk of prostate cancer $(\mathrm{CaP})$.

Initial mutation screening of $E L A C 2$ in prostate cancer patients from Utah (Tavtigian et al. 2000, 2001) revealed four nucleotide sequence variants that altered the predicted amino acid sequence of the protein. Two of these variants, the frameshift mutation 1641insG and the missense variant Arg781His, are rare or pedigree-specific changes associated 
with a high risk of $\mathrm{CaP}$. The other two variants are common missense variants: Ser217Leu (S217L), with a Leu217 allele frequency of about $30 \%$ in Caucasians of northern European ancestry, and Ala541Thr (A541T), with a Thr541 allele frequency of about $5 \%$ in the same population. Two primary hypotheses emerged from the initial case-control association study to examine these common missense changes (Tavtigian et al. 2001): (1) Thr541 is in very strong linkage disequilibrium with Leu217 such that virtually all Thr541 carriers also carry Leu217. If confirmed, the two missense variants could be considered to define a threeallele system: Ser217/Ala541 (SA allele), Leu217/Ala541 (LA allele), and Leu217/Thr541 (LT allele). (2) Both Leu217 and Thr541 confer a modestly increased risk of $\mathrm{CaP}$, with the risk of prostate cancer conferred by the individual alleles decreasing in the order $\mathrm{LT}>\mathrm{LA}>\mathrm{SA}$.

Subsequent studies have both screened familial prostate cancer patients for germline mutations in ELAC2 (Xu et al. 2001; Rokman et al. 2001; Wang et al. 2001) and looked for associations between common sequence variants in this gene and CaP-related phenotypes (Xu et al. 2001; Rokman et al. 2001; Wang et al. 2001; Rebbeck et al. 2000; Vesprini et al. 2001; Suarez et al. 2001). Rare novel sequence variants that alter the protein coding sequence of ELAC2 have been found in familial CaP patients (Rokman et al. 2001; Wang et al. 2001). However, as these resequencing studies have focused on variant discovery in $\mathrm{CaP}$ patients without measuring the summed frequency of such variants in controls, it is not known whether such variants are actually more common in patients than in controls. In addition, the variants that have been found are less than $100 \%$ penetrant and do not account for all of the cases in the pedigrees in which they are present, making it difficult to link them with risk of disease. While association studies have confirmed strong linkage disequilibrium between the common missense variants Thr541 and Leu217, they have left the relationship between carriage of these common ELAC2 missense variants and risk of $\mathrm{CaP}$ ambiguous. For instance, Rebbeck et al. (2000) observed an association between carriage of the LT allele of ELAC2 and risk of $\mathrm{CaP}$ in a hospital-based $\mathrm{CaP}$ case-control series, and Suarez et al. observed an association between carriage of the LT allele of ELAC2 and risk of $\mathrm{CaP}$ in a series of familial $\mathrm{CaP}$ cases vs. low-risk controls (Suarez et al. 2001). In contrast, other studies have seen no significant evidence for an association between the common missense variants in ELAC2 and risk of CaP (Xu et al. 2001; Rokman et al. 2001; Wang et al. 2001; Vesprini et al. 2001).

Age-adjusted incidence rates of $\mathrm{CaP}$ vary dramatically both by geographic location and by ethnicity (Muir et al. 1987; Parkin et al. 1992; Kolonel 1996). While some incidence-rate differences between ethnic groups may be due to allele-frequency differences for modest risk sequence variants (Shibata et al. 1997; Makridakis et al. 1997; Walker et al. 1998), so little is understood about the genetic component of prostate cancer susceptibility that the magnitude of this effect cannot yet be estimated. One consistent pattern is that Asian men residing in Asia experience considerably lower $\mathrm{CaP}$ incidence and mortality rates than Caucasians living in the United States. However, CaP inci- dence among first- and second-generation Asian Americans is substantially higher than that in men living in Asia, sometimes approaching the rate in Caucasians (Kolonel 1996; Cook et al. 1999). As this immigration effect was well recognized before prostate-specific antigen (PSA) screening came into common use and is only partially accounted for by differences in screening and/or reporting (Cook et al. 1999; Shibata et al. 1997), a large fraction of the difference in prostate cancer incidence between United States Caucasians and Asians residing in Asia is presumably attributable to environmental, lifestyle, and/or dietary differences. Thus, one might expect that the genetic component of susceptibility to $\mathrm{CaP}$ is less confounded by these factors, and consequently easier to detect, in Asian men residing in Asia than in Caucasian men residing in the United States. Therefore, even though point estimates of allele frequencies of the missense variants of ELAC2 are numerically lower in Asians than in Caucasians (Vesprini et al. 2001), we undertook to analyze these variants in a Japanese case-control series.

\section{Subjects and methods}

\section{Study subjects}

Three groups of male Japanese adults were identified for this study.

Prostate cancer cases. This group consisted of 350 patients over the age of 45 who were diagnosed with prostate cancer in Nippon Medical School Hospital, Yokohama City University Hospital, Akita University Hospital, Kyoto University Hospital, Kouchi Medical school Hospital, Nagoya City University Hospital, or Chiba University Hospital over the period 1995-2001. All of the patients had prostectomy with postoperative pathological validation of the diagnosis; however, information on age at diagnosis, tumor stage, and tumor grade were available only for subsets of patients, as follows: age at diagnosis, 255 (72.9\%). The mean age at diagnosis was 66.2 (range of 45-90). Tumor stage, scored by the Jewett staging system, where stages A and B describe locally confined prostate tumors, stage $\mathrm{C}$ describes tumors that have spread across the prostatic capsule or involve the seminal vesicles but without evidence of more distant metastasis, and stage $\mathrm{D}$ describes node-positive tumors and tumors that have metastasized to distant sites, 295 (84.3\%); tumor grade, by Gleason score (range of 2-10), 257 (73.4\%); tumor grade, by a summary description of well differentiated, moderately differentiated, or poorly differentiated, 177 (50.6\%). These clinical data are summarized in Table 1.

Low-risk controls. This group consisted off 114 adult male Japanese with a mean age of 64.5 (52-78), who were negative for prostate cancer after urological examination, which included digital rectal examination (DRE) and rectal ultrasound echography, and had normal serum PSA levels (less than $4 \mathrm{ng} / \mathrm{ml}$ ). 
Table 1. ELAC2 genotype by clinical characteristics in prostate cancer patients $(\mathrm{CaP})$

\begin{tabular}{|c|c|c|c|c|c|}
\hline & Count & SA/SA & Any Leu 217 & Carry LA & Carry LT \\
\hline Age at diagnosis & & & Age & & \\
\hline$<55$ & 14 & 14 & 0 & 0 & 0 \\
\hline $55-59$ & 20 & 18 & 2 & 2 & 0 \\
\hline 60-64 & 60 & 56 & 4 & 3 & 1 \\
\hline $65-69$ & 100 & 95 & 5 & 3 & 2 \\
\hline $70-74$ & 37 & 34 & 3 & 2 & 1 \\
\hline$\geqslant 75$ & 24 & 22 & 2 & 2 & 1 \\
\hline Total & 255 & 239 & 16 & 12 & 5 \\
\hline \multicolumn{6}{|l|}{ Age tertiles } \\
\hline $45-63$ & 78 & 73 & 5 & 4 & 1 \\
\hline 64-68 & 90 & 86 & 4 & 2 & 2 \\
\hline 69-90 & 87 & 80 & 7 & 6 & 2 \\
\hline Total & 255 & 239 & 16 & 12 & 5 \\
\hline Jewitt stage & \multicolumn{5}{|c|}{ Stage } \\
\hline A & 8 & 8 & 0 & 0 & 0 \\
\hline B & 127 & 119 & 8 & 5 & 3 \\
\hline $\mathrm{C}$ & 84 & 77 & 7 & 6 & 2 \\
\hline $\mathrm{D}$ & 76 & 72 & 4 & 3 & 1 \\
\hline Total & 295 & 276 & 19 & 14 & 6 \\
\hline Gleason score & \multicolumn{5}{|c|}{ Grade } \\
\hline 2 & 1 & 1 & 0 & 0 & 0 \\
\hline 3 & 18 & 18 & 0 & 0 & 0 \\
\hline 4 & 16 & 15 & 1 & 0 & 0 \\
\hline 5 & 43 & 41 & 2 & 1 & 1 \\
\hline 6 & 35 & 35 & 0 & 0 & 0 \\
\hline 7 & 76 & 67 & 9 & 7 & 2 \\
\hline 8 & 19 & 18 & 1 & 0 & 1 \\
\hline 9 & 39 & 37 & 2 & 0 & 0 \\
\hline 10 & 10 & 10 & 0 & 0 & 0 \\
\hline Total & 257 & 242 & 15 & 8 & 4 \\
\hline \multicolumn{6}{|c|}{ Categories $-\mathrm{W} / \mathrm{M} / \mathrm{P}$} \\
\hline Well & 35 & 33 & 2 & 1 & 1 \\
\hline Moderate & 99 & 94 & 5 & 4 & 1 \\
\hline Poor & 43 & 39 & 4 & 3 & 1 \\
\hline Total & 177 & 166 & 11 & 8 & 3 \\
\hline
\end{tabular}

SA, Ser217/Ala541 allele; LA, Leu217/Ala541 allele; LT, Leu217/Thr541 allele; W/M/P, Well/Moderately/Poorly differentiated

Unscreened controls. This group consisted of 242 adult male Japanese volunteers with a mean age of 62.8 (49-73). Serum PSA levels and past or present cancer history were unavailable from this group. The case and control series were recruited from patients in the Nippon Medical School Hospital, Yokohama City University Hospital, Akita University Hospital, Kyoto University Hospital, Kouchi Medical School Hospital, Nagoya City University Hospital, and Chiba University Hospital. This collaborative project was approved by the central institutional review board of each individual institution. Cases, random controls, and low-risk controls of the Japanese panels were all recruited from the same regional populations through visits to participating academic hospitals; thus, the variation of genetic background in the Japanese subjects was negligible among the sample populations analyzed in the present study.

\section{Genotyping}

DNAs were extracted from peripheral blood lymphocytes by conventional methods. Every sample was genotyped for the S217L and A541T molecular variants of the ELAC2 gene twice, once by polymerase chain reaction-restriction fragment length polymorphism (PCR-RFLP) and once by single-strand conformation polymorphism (SSCP) analysis. For genotyping by PCR-RFLP, ELAC2 exon 7 was amplified as a 296-bp fragment using the forward primer 5'-AGGTGTGCTGATTTAATTGGCG-3' and reverse primer 3'-AGGCAGAGAATTAAGAAAACGCAAG- 
$5^{\prime}$; the S217L polymorphism was genotyped by restriction digest with TaqI. Exon 17 was amplified as a 419-bp PCR product using the forward primer 5'-CCAGCCTTTGTG TAAGTCTAC-3' and reverse primer $3^{\prime}$-GAGACAG AAATTTTCTGCAGTTGCTTC-5' and typed by restriction digest with Fnu4HI. After digestion, the PCR products were separated on $3 \%$ agarose gels and were visualized by UV light after ethidium bromide staining (Rebbeck et al. 2000).

For genotyping by SSCP, exons 7 and 17 were PCRamplified using the above mentioned primers (independently from the PCR reactions of the PCR-RFLP genotyping). From each reaction, about 10 ng of PCR product was heat-denatured in the presence of $80 \%$ deionized formamide and electrophoresed in $8 \%$ polyacrylamide gels, with or without $10 \%$ glycerol, in $0.5 \times$ tris-borate + EDTA) (TBE) at $8 \mathrm{~V} / \mathrm{cm}$ for $10 \mathrm{~h}$ at room temperature. DNA fragments were visualized by silver staining using the Plus One DNA silver staining kit (Pharmacia Biotech, Tokyo, Japan). Each fragment showing an altered SSCP band pattern was subcloned with an AdvanTAge PCR cloning kit
(Clontech, Palo Alto, CA, USA) and sequenced. There were no discrepancies between genotypes determined by the two methods.

\section{Statistical analyses}

A $\chi$-squared analysis was used to test the significance of genotype frequency differences between distinctive study groups. As some cells of the contingency tables in Tables 2 and 3 contained an expected value of less than 5, all $P$ values in Table 3 were calculated by Fisher's Exact Test (FET). The Mantel-Haenszel $\chi$-squared test with continuity correction was used to summarize data from multiple studies. In the contingency table-based frequency comparisons, $P$ values and odds ratios for LT carriers were calculated by two different methods: (1) A full analysis wherein SA/SA, LA/SA, and LA/LA (i.e., non-LT) genotype carriers were grouped into the reference genotype category; and (2) a reference analysis wherein only SA/SA genotype carriers were used as the reference genotype category.

Table 2. Joint genotype distributions of the Ser217Leu and Ala541Thr variants in a Japanese case-control series

\begin{tabular}{|c|c|c|c|c|c|c|c|c|c|}
\hline \multirow{4}{*}{$\begin{array}{l}\text { Residue } 217 \\
\text { SS }\end{array}$} & \multicolumn{9}{|c|}{ Residue 541} \\
\hline & \multicolumn{3}{|c|}{ Low PSA controls } & \multicolumn{3}{|c|}{ Unscreened controls } & \multicolumn{3}{|l|}{ Cases } \\
\hline & AA & AT & TT & AA & AT & TT & AA & AT & TT \\
\hline & 114 & - & - & 238 & - & - & 331 & - & - \\
\hline SL & - & - & - & 4 & - & - & 13 & 5 & - \\
\hline LL & - & $\bar{n}=114$ & - & - & $\bar{n}=242$ & - & - & 1 & - \\
\hline Allele Freq & & & & & & & & & \\
\hline SA & 1.0000 & & & 0.9917 & & & 0.9714 & & \\
\hline LA & 0.0000 & & & 0.008 & & & 0.0200 & & \\
\hline LT & 0.0000 & & & 0.0000 & & & 0.0086 & & \\
\hline
\end{tabular}

A, Ala541; T, Thr541; S, Ser217; L, Leu217; PSA, prostase-specific antigen

Table 3. Association between prostate cancer and the ELAC2 genotype

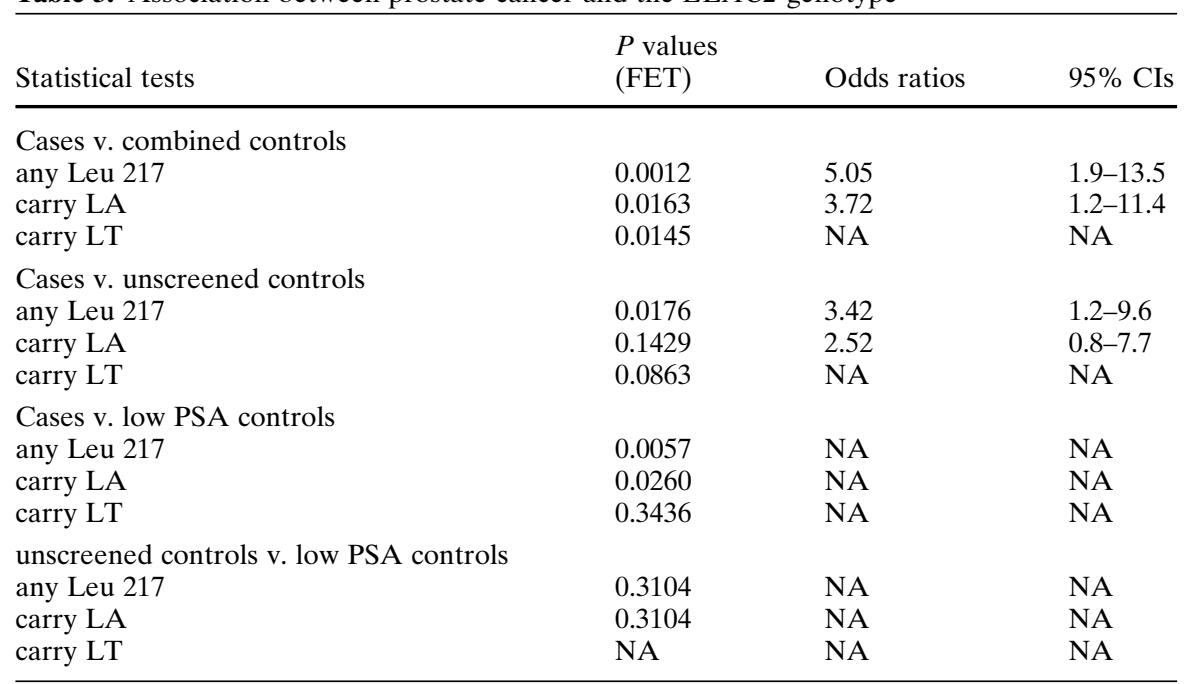

NA, not applicable; FET, Fisher's Exact Test; CIs, confidence intervals; V, versus 
In order to compare the degree of disequilibrium between different pairwise combinations of loci, a frequencyindependent measure of disequilibrium $\left(\mathrm{D}^{\prime}\right.$, the proportion of maximum possible disequilibrium $\left(\mathrm{D}_{\max }\right)$ in the given direction) was calculated, where $\mathrm{D}^{\prime}=\hat{\mathrm{D}} /\left|\mathrm{D}_{\max }\right| . \hat{\mathrm{D}}$ was calculated using the maximum likelihood technique implemented in the program EH (Xie and Ott 1993), and $\hat{\mathrm{D}}=$ $\mathrm{p}(1-\mathrm{q})$ since A541T and S217L are in positive linkage disequilibrium, where $\mathrm{p}$ and $\mathrm{q}$ are the frequencies of the less common allele at each respective variant, and $\mathrm{p}<\mathrm{q}$.

Finally, a trend statistic was calculated by grouping the cases and controls into three genotype classes [SA/SA, (LA/ SA and LA/LA), and (LT/SA \& LT/LA \& LT/LT)] and applying the Cochran-Armitage trend test (Cochran 1954; Armitage 1955) (implemented as a summary test analogous to the Mantel-Haenszel $\chi$-squared test) using a simple linear trend $(0,1,2)$ for the row scores.

\section{Results}

Comparison of Ser217Leu and Ala541Thr carrier frequencies between Japanese and Caucasians

Our sample set consisted of 706 adult Japanese men: 350 CaP patients, 114 low-risk controls, and 242 men from the general population. All patients and controls were genotyped at S217L and A541T by both PCR-RFLP and SSCP. Genotypes were recorded at both markers for all samples. Genotyping revealed that both Leu217 and Thr541 are present in the Japanese population, but at dramatically lower frequencies than in the Caucasian population samples studied to date. Comparison of all the Japanese men (cases and controls) with a series of Caucasian (European ancestry) low-risk controls (Suarez et al. 2001) is illustrative (Table 4). Carrier frequencies for the Leu217 variant were $3.3 \%$ in Japanese versus $46.5 \%$ in Caucasian controls $(P<$ $\left.1 \times 10^{-5}\right)$. Carrier frequencies for the Thr541 variant were $0.8 \%$ in Japanese versus $3.7 \%$ in the Caucasian controls $(P$ $=0.001)$. As carrier frequencies in the Caucasian controls used for this comparison are among the lowest for any
Caucasian series so far examined, it is clear that the frequencies of these two variants are much lower in the Japanese than in the Caucasian population.

\section{Disequilibrium between Thr541 and Leu217}

We observed six Thr541 carriers, all heterozygotes, in our set of 706 Japanese men. Each of the Thr541 carriers also carried Leu217 (Table 4); thus, we observed strong linkage disequilibrium between the two variants, and the maximum likelihood frequency estimate for the LT allele was the same as that for Thr541. As previous studies have also observed near-complete linkage disequilibrium between these two variants, the two missense changes can indeed be considered to define a three-allele (SA, LA, LT) system.

\section{Case-control comparison}

The study format allowed for three case-control comparisons (with the controls pooled or with the controls divided into low-risk and population series), as well as a comparison between unscreened controls and low-risk controls (Tables 2, 3). In order to distinguish the effect of carrying Leu217 from that of carrying Leu217 and Thr541, Leu217 carriers were analyzed both as a group and broken into the subsets LA carriers and LT carriers. One patient had the genotype LA/LT and was therefore analyzed in both subsets. Prostate cancer patients were significantly more likely to carry Leu217 than controls $(P=0.001)$. This was true whether they were compared with the low-risk controls $(P=0.006)$ or the unscreened controls $(P=0.018)$. The odds ratio (OR) in the overall case-control comparison, $5.05[95 \%$ confidence interval (CI) 1.9-13.5], was much higher than those reported in previous case-control analyses of the Leu217 variant from primarily Caucasian populations, with even the lower bound of the $95 \%$ confidence interval higher than the OR reported in previous studies. When Leu217 carriers were broken down into the subsets of LA carriers and LT carriers, the frequency of LA carriers was higher in the patients than in the controls $(P=0.016$; OR $=3.72$;

Table 4. Joint genotype distributions of the Ser217Leu and Ala541Thr variants in a sample of Japanese prostate cancer patients and controls compared with a Caucasian prostate cancercontrol series

\begin{tabular}{|c|c|c|c|c|c|c|}
\hline \multirow[b]{3}{*}{ Residue 217} & \multicolumn{3}{|c|}{ Residue 541} & & & \\
\hline & \multicolumn{3}{|c|}{ Japanese series } & \multicolumn{3}{|c|}{ Caucasian series } \\
\hline & AA & AT & TT & AA & AT & TT \\
\hline SS & 683 & - & - & 190 & - & - \\
\hline SL & 17 & 5 & - & 124 & 10 & - \\
\hline LL & - & $\begin{array}{l}1 \\
n=706\end{array}$ & - & 28 & $\begin{array}{l}1 \\
n=355\end{array}$ & 2 \\
\hline \multicolumn{7}{|l|}{ Allele Freq } \\
\hline SA & 0.9830 & & & 0.7239 & & \\
\hline LA & 0.0127 & & & 0.2549 & & \\
\hline LT & 0.0042 & & & 0.0211 & & \\
\hline
\end{tabular}

data series from Suarez et al. (2001) 
95\% CI, 1.2-11.4). Even when the one LA/LT heterozygote was excluded from the analysis, the frequency of LA carriers was higher in patients than in controls $(P=0.027$; OR $=$ 3.46 ; 1.12-10.71), providing strong evidence that, in this population, carriage of Leu217 conferred increased risk of prostate cancer independent of genotype at Thr541.

Interestingly, none of the 356 controls carried Thr541, compared with 6 of the 350 cases. Cases were significantly more likely to carry Thr541, and therefore the LT allele, than controls $(P=0.015)$. In order to estimate an odds ratio for carriage of LT, we added a count of 0.5 to each cell in the contingency table. The estimated odds ratio of 9.7 was higher than that calculated for Leu217 carriers, as expected from the observation that the OR for Leu217 carriers was higher than that for LA carriers. This OR is also higher than that reported in any case-control analysis of the Thr541 variant in primarily Caucasian populations. None of the 114 low risk controls carried either Leu217 or Thr541. However, the carrier frequency difference between the low-risk controls and the unscreened controls was not statistically significant $(P=0.310)$.

\section{Stratified analysis}

Cases were stratified by age at diagnosis $(<55,55-59,60-64$, $65-69,70-74$, and $\geq 75$ years), age at diagnosis tertiles (4563, 64-68, and 69-90 years), tumor stage (Jewitt stages A, B, $\mathrm{C}$, or D), tumor grade (Gleason scores 2-10), and tumor grade categories (Gleason scores $2-4,5-7$, or 8-10). Table 1 shows the distribution of the ELAC2 genotype by each clinical characteristic. There were no statistically significant or even borderline carrier frequency differences between any of the case strata and ELAC2 genotype.

\section{Discussion}

The strong linkage disequilibrium between the ELAC2 missense variants Thr541 and Leu217 observed in Caucasian population samples is also maintained in the Japanese population. The variants therefore define three common alleles, SA, LA, and LT. The case-control data are consistent with the hypothesis that both Leu217-containing alleles confer risk of $\mathrm{CaP}$, and the risk conferred decreases in the order LT > LA > SA. The potential fourth allele, Ser217/ Thr541 (ST allele), is apparently so rare that it may not be possible to address its relationship with $\mathrm{CaP}$ risk in a casecontrol format.

In order to understand how the data reported here fit into the context of the ambiguous results reported from North American and European studies of this gene, we performed a pooled analysis of six of those studies (Table 5). If the case-control series in those studies are classified by two binary variables (familial vs. unselected patients; lowrisk vs. unscreened controls) and then analyzed in the four resultant categories, several clear trends emerge. When the analysis is limited to the two studies that compare familial cases to stringently low-risk controls (Xu et al. 2001; Suarez

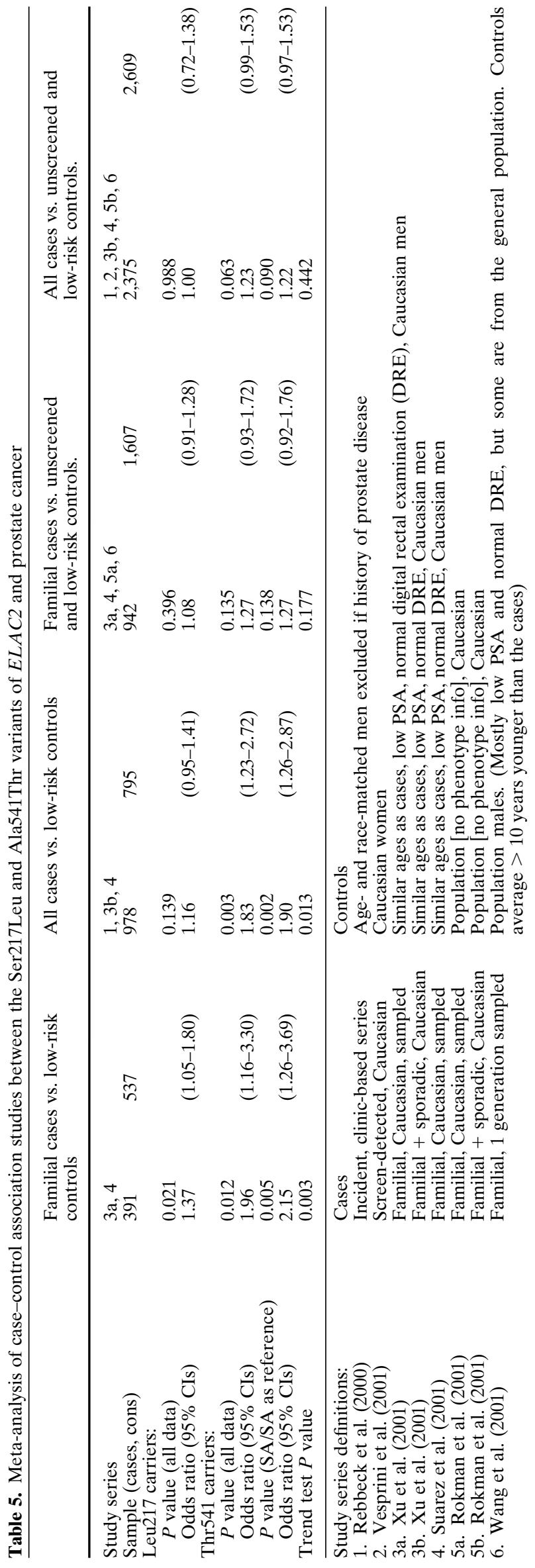


et al. 2001), cases are more likely to be Leu217 carriers than controls $(P=0.021 ; \mathrm{OR}=1.37 ; 1.05-1.80)$ and are also more likely to be LT carriers than controls $(P=0.005$; OR $=2.15 ; 1.26-3.69)$. Assuming $100 \% \mathrm{D}^{\prime}$ linkage disequilibrium between the two missense variants (none of these cohorts contained any Thr541 carriers who were not Leu217 carriers), we find a significant trend $(P=0.003)$ for genotype-conferred risk in the order (LT/SA or LT/LA or $\mathrm{LT} / \mathrm{LT})>(\mathrm{LA} / \mathrm{SA}$ or LA/LA $)>\mathrm{SA} / \mathrm{SA}$. Thus, these results are qualitatively identical to those from the Japanese cohort reported here, albeit at considerably lower odds ratios. Two additional case-control series compare unselected cases with low-risk controls (Xu et al. 2001; Rebbeck et al. 2000). When these case and control series are added into the analysis, evidence that Leu217 carriers are at increased risk is no longer significant $(P=0.139$; OR $=1.16$; 0.95 1.41). However, evidence for a higher frequency of Thr541 carriers in cases than in controls is strengthened $(P=0.002 ;$ OR $=1.9 ; 1.26-2.87)$. In contrast, addition of the three case-control series containing unscreened controls (Rokman et al. 2001; Wang et al. 2001; Vesprini et al. 2001) to the analysis completely eliminates evidence that Leu217 carriers are at increased risk $(\mathrm{OR}=1.00 ; 0.72-1.38)$ and leaves only marginal evidence that Thr541 carriers are at increased risk $(P=0.063$ or 0.090 , depending on whether calculated by full or reference method, respectively; OR = $1.2 ; 1.0-1.5)$.

The pooled analysis is more powerful than any of the individual studies that it samples because the number of cases and controls subsumed in each category is much larger than in the individual component studies. In particular, when familial $\mathrm{CaP}$ cases are analyzed versus low-risk controls, certain marginal results or trends reported in the individual studies become statistically significant. From the pooled analysis, we draw two conclusions: (1) In North American or European Caucasians, the LA and LT alleles of $E L A C 2$ confer greater risk in the context of familial than sporadic $\mathrm{CaP} ;(2)$ There is a distinct difference between use of unscreened controls and age-matched low-risk controls in these case-control association studies. In support of this latter conclusion, Rokman et al. (2001) reported a higher frequency of LT carriers among men with benign prostate hyperplasia (BPH) than among unscreened controls, and Vesprini et al. (2001) noted a trend toward higher LT carrier frequency in men with elevated PSA and BPH than in men with elevated PSA but normal prostate biopsy.

It is not obvious that any simple explanation can entirely resolve the apparent differences between the data sets reported here. While it is reassuring that the Japanese casecontrol and Caucasian familial case versus low-risk control data sets are qualitatively similar, the basis of the quantitative difference between them remains unclear. One possibility is that diet and lifestyle factors in North America and Europe are responsible for a sufficient fraction of $\mathrm{CaP}$ risk in these geographical regions that they confound detection of the risk that is conferred by the LA and LT alleles of $E L A C 2$. On the other hand, Japanese men residing in Japan may be subject to gene-environment or gene-gene interactions that magnify risk associated with those alleles.
Analysis of genotyping data from Japanese men residing in North America or Europe could potentially clarify this issue, subject to the limitation that the study would have to be sufficiently powerful to detect an odds ratio of about 1.5 at a carrier frequency of about $1 \%$. The difference in the findings between studies utilizing low-risk controls as opposed to unscreened controls is also somewhat puzzling. It may be that the LA and LT alleles of ELAC2 predispose patients to both malignant and nonmalignant prostate disease in such a way that the use of unscreened controls confounds the analysis. Alternatively, these alleles could confer risk of nonmalignant prostatic phenotypes, such as $\mathrm{BPH}$, for which men often seek medical evaluation; coupled with the high rate of occult prostate cancer in older men, this could lead to a true association with BPH and an ascertainment bias-driven association with prostate cancer. Such issues underscore the point that the power of case-control association studies of modest risk sequence variants is very sensitive to case and control category definitions. The optimally designed study would define phenotype categories that are related to or can confound the main disease category and consider such categories separately. Using these phenotypic characteristics, such a study would also define a category of controls at lower risk than the general population.

In summary, we found a significantly higher frequency of both the LA and LT alleles of ELAC2 in unselected Japanese CaP patients compared with Japanese male controls, with evidence that the $\mathrm{CaP}$ risk conferred by the missense-defined alleles of this gene decrease in the order LT $>$ LA $>$ SA. Comparison of these data with a pooled analysis of studies utilizing Caucasian $\mathrm{CaP}$ patients and low-risk controls revealed results that are qualitatively very similar and therefore confirmatory, although the odds ratios that we calculated from the Japanese case-control series are considerably higher than those derived from the Caucasian series. While these data provide strong evidence that sequence variants in $E L A C 2$ can confer increased risk of $\mathrm{CaP}$, we can only speculate as to why the magnitude of this effect differs between ethnic groups.

Acknowledgments This work was supported in part by NIH Grant CA90752-01 (to N.J.C.), by a grant-in-aid for the Scientific Research priority area of "Cancer" from the Ministry of Education, Culture, Science and Technology of Japan, and by a Research for the Future Program Grant of The Japan Society for the Promotion of Science. We thank Ms. Kyoko Shimizu, Mayumi Tanaka, and Naoko Tsuruta for their contributions. We also thank Julia Reid and James Farnham for helpful suggestions and Kate Nathanson for a critical reading of the manuscript.

\section{References}

Armitage P (1955) Tests for linear trends in proportions and frequencies. Biometrics 11:375-386

Cochran WG (1954) Some methods of strengthening the common chi-squared tests. Biometrics 10:417-451

Cook LS, Goldoft M, Schwartz SM, Weiss NS (1999) Incidence of adenocarcinoma of the prostate in Asian immigrants to the United States and their descendants. J Urol 161:152-155 
Kolonel LN (1996) Racial and geographic variations in prostate cancer and the effect of migration. In: Fortner JG, Sharp PA (eds) Accomplishments in cancer research Lippincott-Raven, Philadelphia, pp 221-228

Makridakis N, Ross RK, Pike MC, Chang L, Stanczyk FZ, Kolonel LN, Shi CY, Yu MC, Henderson BE, Reichardt JK (1997) A prevalent missense substitution that modulates activity of prostatic steroid 5alpha-reductase. Cancer Res 57:1020-1022

Muir C, Waterhouse J, Mack T, Powell J, Whelan S (1987) Cancer Incidence in five continents, Vol. 5. International Agency for Research on Cancer, Lyon, France

Parkin DM, Muir C, Whelan S (1992) Cancer incidence in five continents, Vol. 6. International Agency for Research on Cancer, Lyon, France

Rebbeck TR, Walker AH, Zeigler-Johnson C, Weisburg S, Martin AM, Nathanson KL, Wein AJ, Malkowicz SB (2000) Association of HPC2/ELAC2 genotypes and prostate cancer. Am J Hum Genet 67:1014-1019

Rokman A, Ikonen T, Mononen N, Autio V, Matikainen MP, Koivisto PA, Tammela TL, Kallioniemi OP, Schleutker J (2001) ELAC2/ $H P C 2$ involvment in hereditary and sporadic prostate cancer. Cancer Res 61:6038-6041

Shibata A, Whittemore AS, Imai K, Kolonel LN, Wu AH, John EM, Stamey TA, Paffenbarger RS (1997) Serum levels of prostatespecific antigen among Japanese-American and native Japanese men. J Natl Cancer Inst 89:1716-1720

Suarez BK, Gerhard DS, Lin J, Haberer B, Nguyen L, Kesterson NK, Catalona WJ (2001) Polymorphisms in the prostate cancer susceptibility gene $H P C 2 / E L A C 2$ in multiplex families and healthy controls. Cancer Res 61:4982-4984

Tavtigian SV, Simard J, Labrie J, Skolnick MH, Neuhausen SL, Rommens J, Cannon-Albright LA (2000) A strong candidate pros- tate cancer predisposition gene at chromosome 17p. Am J Hum Genet suppl 67:11

Tavtigian SV, Simard J, Teng DH, Abtin V, Baumgard M, Beck A, Camp NJ, Carillo AR, Chen Y, Dayananth P, Desrochers M, Dumont M, Farnham JM, Frank D, Frye C, Ghaffari S, Gupte JS, Hu R, Iliev D, Janecki T, Kort EN, Laity KE, Leavitt A, Leblanc G, McArthur-Morrison J, Pederson A, Penn B, Peterson KT, Reid JE, Richards S, Schroeder M, Smith, R, Snyder SC, Swedlund B, Swensen J, Thomas A, Tranchant M, Woodland AM, Labrie F, Skolnick MH, Neuhausen S, Rommens J, Cannon-Albright LA (2001) A candidate prostate cancer susceptibility gene at chromosome $17 \mathrm{p}$. Nat Genet 27:172-180

Vesprini D, Nam RK, Trachtenberg J, Jewett MA, Tavtigian SV, Emami M, Ho M, Toi A, Narod SA (2001) HPC2 variants and screen-detected prostate cancer. Am J Hum Genet 68:912-917

Walker AH, Jaffe JM, Gunasegaram S, Cummings SA, Huang CS, Chern HD, Olopade OI, Weber BL, Rebbeck TR (1998) Characterization of an allelic variant in the nifedipine-specific element of CYP3A4: ethnic distribution and implications for prostate cancer risk. Mutations in brief no. 191 (Online). Hum Mutat 12:289

Wang L, McDonnell SK, Elkins DA, Slager SL, Christensen E, Marks AF, Cunningham JM, Peterson BJ, Jacobsen SJ, Cerhan JR, Blute ML, Schaid DJ, Thibodeau SN (2001) Role of HPC2/ELAC2 in hereditary prostate cancer. Cancer Res 61:6494-6499

Xie X , Ott J (1993) Testing linkage disequilibrium between a disease gene and marker loci. Am J Hum Genet suppl 53:1107

$\mathrm{Xu}$ J, Zheng SL, Carpten JD, Nupponen NN, Robbins CM, Mestre J, Moses TY, Faith DA, Kelly BD, Isaacs SD, Wiley KE, Ewing CM, Bujnovszky P, Chang B, Bailey-Wilson J, Bleecker ER, Walsh PC, Trent JM, Meyers DA, Isaacs WB (2001) Evaluation of linkage and association of $H P C 2 / E L A C 2$ in patients with familial or sporadic prostate cancer. Am J Hum Genet 68:901-911 